\title{
Confinement Following Child Birth and Associated Postpartum Mental Distress
}

\author{
Lonia Mwape, Mutinta C. Muleya, Patricia Katowa Mukwato, Margaret Maimbolwa \\ School of Nursing Sciences, University of Zambia, Lusaka, Zambia \\ Email: loniamagolo@gmail.com
}

How to cite this paper: Mwape, L., Muleya, M.C., Mukwato, P.K. and Maimbolwa, M. (2018) Confinement Following Child Birth and Associated Postpartum Mental Distress. Open Journal of Psychiatry, 8, 152-167. https://doi.org/10.4236/ojpsych.2018.82015

Received: March 27, 2018

Accepted: April 25, 2018

Published: April 28, 2018

Copyright (C) 2018 by authors and Scientific Research Publishing Inc. This work is licensed under the Creative Commons Attribution International License (CC BY 4.0).

http://creativecommons.org/licenses/by/4.0/ (c) (i) Open Access

\begin{abstract}
Objective: This study sought to explore confinement following childbirth and its association with mental distress. Methods: A descriptive-analytical cross sectional study design was used. The study was conducted in two districts of Zambia representing an urban and rural setting, targeting parenting mothers during the first two months after delivery. Systematic random sampling technique was used to select the participants. Every second case seen at the study sites between March to August 2017 was selected to participate in the study. The data were collected using the Edinburgh Postnatal Depression Scale and analyzed using the Statistical Package for Social Sciences (SPSS) version 22. Pearson correlation coefficient was used to determine the relationship between the dependent and independent variables while binary logistic regression was used to check the combined impact of independent variables on mental distress. Ethical approval was obtained from the local Research Ethics Committees. Participation in the study was voluntary. Results: A total of 200 women participated in the study. The results revealed a high prevalence of mental distress, with 42 percent of women reporting severe levels, and 36.0 percent showing moderate levels. A statistically significant relationship was found between mental distress and confinement $\left(X^{2}=29.289 ; \mathrm{P}=0.001\right)$, residential type $\left(X^{2}=16.863 ; P=0.001\right)$, and age $\left(X^{2}=17.996 ; P=0.006\right)$. Suicidal thoughts during confinement were also found to be prevalent in 16 percent of the participants. Binary logistic regression revealed that changes in confinement period from below 2 weeks to between 2 weeks and 1 month raised the odds of suffering mental distress 5.127 times while changes from a confinement period of between 2 weeks and 1 month to over 1 month raised the odds of suffering mental distress 7.737 times. Conclusion: The study results confirmed an association between confinement and mental distress, revealing that the longer the confinement, the more at risk the women were of mental distress.
\end{abstract}




\section{Keywords}

Mental Distress, Confinement, Childbirth, Postnatal, Postpartum

\section{Introduction}

The postnatal period is the time starting immediately following delivery, and lasting up to about six weeks post-delivery. It is considered one of the most life threatening [1], [2], yet overlooked phases in the lives of mothers and babies as most deaths have been reported to occur during this period [2].

Many societies in the world have specific practices applicable to a woman in the few days that follow childbirth. Postpartum confinement is one of the traditional practices observed by women following childbirth, in some cultures. It is defined as a traditional practice meant to facilitate recovery of a new mother from the process of childbirth [3]. In this paper, postpartum confinement is described as a custom practiced by some tribes in Zambia, and involves seclusion of a new mother within the house where the mother is exposed to some prescribed rituals. These postnatal rituals are meant to avoid future ailments for the mother and the baby in some cultures, while in others, they are meant to facilitate rest for the mother. They are believed to contribute favourably to the positive health outcomes [4].

When women are in confinement, certain restrictions are imposed on them, which may include, but not limited to dietary, hygiene practices and participating in home chores [5], [4]. According to Okka et al. [1], confining the mother and her baby to the house for about 40 days is one of the most common practices in Turkey, and despite the availability of contemporary maternal health services in general and postnatal health services in particular, traditional practices are still being adhered to alongside contemporary health services [1].

Further, Cousik and Hickey [6] highlight that older women in communities which observe maternal practices are mandated to ensure traditional rules set for pregnant and postnatal women are adhered to. The older women are considered the main sources of traditional knowledge as they model for the younger generation by following traditional practices themselves. The uptake of the modelled knowledge facilitates group identity and empowers women with skills to disseminate the same knowledge to others. As earlier indicated, included among the laid down rules is postpartum confinement, which may last up to about three weeks [6].

It follows therefore, that postnatal confinement, when examined from a positive perspective, serves as an enriched social support strategy, (even though a woman is labeled as contaminated in some settings), and may help limit likelihood of developing mental distress. While it is recognized that postnatal confinement protects women against postnatal mental distress, Demirel et al. [4] argue that observing some of the traditional practices predisposes women to 
psychological distress arising from the women's compromised self esteem and limited freedom.

Chien et al., [7] writing from Taiwan assert that observance of the traditional practices during postpartum confinement accelerates better health for postpartum mothers. Similarly, Chen et al., [8] found that conformity to confinement and rituals in imposed during confinement was negatively associated with postpartum mental distress (OR $=0.93,95 \%$ CI: 0.90 - 0.96).

Conversely, Leung et al. [9] found a positive correlation between confinement and depression and therefore argued that postpartum confinement (doing-the-month) does not actually protect women from postpartum mental distress. In the same line, Liu et al., [10] using the Edinburgh Postnatal Depression Scale (EPDS) found an increase in psychological distress during postpartum confinement. They oppose the notion that adherence to confinement shields women from emotional distress, and emphasize the need for routine screening for postpartum depression during the confinement period.

In addition, Abdollahi et al., [11] attribute postpartum depression to lack of decision making powers by women in confinement, and constrained relationships with caregivers, which deny them the choice to or not to accept the traditional practices and rituals imposed on them. Some components of these traditional practices, as well as conflicts between players have been reported to lead to mental illness.

Taiwan has seen the transformation and shifting of postpartum traditional confinement and practices from homes to health facilities in an effort to minimize the mental distress that may arise from some of the traditional practices. These facilities maintain the "good" traditional practices, and the "bad" ones are modified and challenged while providing justifications for the modifications [12].

There are widespread myths implicating hormonal changes as responsible for the psychological distress experienced by women during the postnatal period, but repeated studies have not linked hormonal changes with postpartum psychological distress [13]. It is suggested that these may be symptoms of pre-existing mental illness, which may be worsened by tiredness, changes in schedule, and stress resulting from the parenting role [14].

Therefore, ensuring good mental health for women in postnatal confinement is critical, especially given that postnatal depression has been found to be associated with increased risk of psychological and developmental disturbances in children and can have adverse effects on the family as well [15], [16]. Mental health problems in general are among the most common morbidities of the perinatal period. Although the phenomenology and risk factors for perinatal mental disorders are largely similar to those for the disorders at other times, treatment considerations may differ during pregnancy and breastfeeding [15].

This study explored confinement following childbirth and its association with mental distress. It is envisaged that the results will inform policy and planning 
for interventions and preventive strategies for mental distress experienced by women in confinement following child birth.

\section{Methodology}

\subsection{Participants and Procedure}

A descriptive-analytical cross sectional study design was used to explore the prevalence of postnatal psychological distress and its association with postpartum confinement. The study was conducted from March to August 2017 and study sites included Chipata and Kalomo Districts of Eastern and Southern Provinces of Zambia, representing an urban and rural setting respectively. Postnatal mothers during the first two months after delivery formed the study population and were accessed from postnatal clinics in the selected health facilities of the two districts. Women in the first two months of the postnatal period were targeted because confinement takes place within that period after delivery. Ten (10) health centres in each of the districts were selected to participate in the study. Purposive sampling technique was used to select participating health facilities, targeting those health facilities with a high number of pregnancies. On the other hand, systematic random sampling technique [17] was used to select the participants. During this process, every $2^{\text {nd }}$ case seen at the study sites between March to August 2017 was selected to participate in the study. The sampling interval was established by dividing 358 (the accessible number of postnatal women) by 200 (the sample size) giving an interval of every $2^{\text {nd }}$ person. The sample size was calculated using Lameshaw et al's [18] formula.

\subsection{Materials}

Women attending postnatal clinics on the day of the interviews were recruited to participate in the study. Recruitment involved getting permission from the Clinic in-charge who helped to identify eligible participants and thereafter, individual participants were invited to participate in the interviews. Participation was voluntary. Assent was obtained from guardians of the participants who were below the legal age of consenting. The Edinburgh Postnatal Depression Scale was the tool used to collect the data that assessed the extent to which women experience mental distress during postpartum confinement. The Edinburgh Postnatal Depression Scale [19], [20] is a ten item self-report screening tool for depression that has been used widely postnatally and has, in the recent years been validated for use antenatally. The scale consists of different domains whose scores are totalled up in order to determine the presence of mental distress or depression.

\subsection{Statistical Analysis}

The data were analyzed using the Statistical Package for Social Sciences (SPSS) version 22. The domains of the Edinburgh Postnatal Depression Scale were computed to get a total score which was then correlated with confinement. Each 
domain was then individually correlated with confinement. Pearson correlation coefficient was used to determine the relationship between confinement following childbirth and postnatal distress, and anxiety and suicidal thoughts. A binary logistic regression test was done to check the combined impact of region, age, education, marital status, parity and confinement on mental distress.

\section{Results}

Participation in the study was based on informed consent. The right to withdraw from participation whenever participants felt like, was upheld throughout the data collection processes.

Table 1 shows that most of the respondents (33\%) were between the ages 15 20 while a small number (17\%) were above 33 years. Most of the respondents (50\%) had attained secondary level education with a small number (9\%) having attained tertiary level education and most respondents (81\%) were married, compared to 12 percent who were single, while 9 percent were divorced.

\subsection{Risk Factors for Postnatal Mental Distress}

The table (Table 2) shows that 48.5 percent of the respondents reported having been confined for more than one month following childbirth while a small number $(11 \%)$ reported being confined for less than two weeks.

Table 1. Frequencies-socio-demographic characteristics.

\begin{tabular}{|c|c|c|}
\hline & Frequency & Percentage \\
\hline \multicolumn{3}{|c|}{ Age } \\
\hline $15-20$ & 66 & 33.0 \\
\hline $21-26$ & 46 & 23.0 \\
\hline $27-32$ & 54 & 27.0 \\
\hline 33 and above & 34 & 17.0 \\
\hline Total & 200 & 100.0 \\
\hline \multicolumn{3}{|c|}{ Level of Education } \\
\hline Primary & 90 & 45.0 \\
\hline Secondary & 101 & 50.0 \\
\hline Tertiary & 9 & 4.5 \\
\hline Total & 200 & 100.0 \\
\hline \multicolumn{3}{|c|}{ Marital Status } \\
\hline Single & 24 & 12.0 \\
\hline Married & 162 & 81.0 \\
\hline Divorced & 9 & 4.5 \\
\hline Widowed & 5 & 2.5 \\
\hline Total & 200 & 100 \\
\hline
\end{tabular}


Table 2. Frequencies-risk factors for mental distress.

\begin{tabular}{ccc}
\hline & Frequency & Percentage \\
\hline Less than 1 Week & Duration of Confinement & 11.0 \\
2 weeks to 1 Month & 22 & 40.5 \\
Above one Month & 81 & 48.5 \\
Total & 97 & 100.0 \\
& 200 & \\
Mild & Rate of Mental Distress & 21.5 \\
Moderate & 43 & 36.0 \\
Severe (High) & 72 & 42.5 \\
Total & 85 & 100.0 \\
& 200 & 74.5 \\
Never & Suicidal thoughts & 9.5 \\
Hardly ever & 149 & 12.0 \\
Sometimes & 19 & 4.0 \\
Quite often & 24 & 100.0 \\
Total & 8 &
\end{tabular}

Most of the respondents (42.5\%) reported high levels of mental distress during the time of confinement following child birth, compared to 21.5 percent who reported mild levels of mental distress as shown in Table 2.

According to Table 2, 74.5 percent of the respondents had never had suicidal thoughts during confinement while a small number (4.0\%) reported having had suicidal thoughts quite often.

\subsection{Cross Tabulations}

The table (Table 3) shows that 46.7 percent of respondents within the age range of 15 and 20 reported higher levels of mental distress while only 20.6 percent of those aged above 33 years reported higher levels of mental distress. At the same time, 16.7 percent of women between the age 15 and 20 reported low levels of mental distress compared to 26.7 percent of those aged above 33 showing low levels of mental distress. As such, there is no statistically significant relationship found between age and mental distress $\left(\mathrm{X}^{2}=17.996, \mathrm{P}<0.006\right)$.

Table 3 also indicates that 45.6 percent of the respondents who had gone up to primary level of education reported high levels of mental distress compared to 33.3 percent of those who had gone up to tertiary level of education who reported high levels of mental distress. Therefore, no statistically significant relationship was found between mental distress and level of education $\left(\mathrm{X}^{2}=0.904, \mathrm{P}\right.$ $<0.924)$. 
Table 3. Cross tabulations.

\begin{tabular}{|c|c|c|c|c|c|}
\hline \multicolumn{6}{|c|}{ Mental Distress and Age } \\
\hline \multirow{2}{*}{ Age } & \multicolumn{3}{|c|}{ Mental Distress } & \multirow{2}{*}{ Total } & \multirow{2}{*}{ P Value } \\
\hline & Low & Moderate & High & & \\
\hline $15-20$ & $11(16.7)$ & $24(36.4)$ & $31(46.7)$ & $66(33.0)$ & \multirow{3}{*}{$\mathrm{X}^{2}=17.996$} \\
\hline $21-26$ & $14(30.3)$ & $7(15.2)$ & $25(54.3)$ & $46(23.0)$ & \\
\hline $27-33$ & $9(16.7)$ & $23(42.6)$ & $22(40.7)$ & $54(27.0)$ & \\
\hline 33 and above & $9(26.7)$ & $18(52.9)$ & $7(20.6)$ & $34(17 . .0)$ & \multirow[t]{2}{*}{$\mathrm{P}<0.006$} \\
\hline Total & $43(21.5)$ & $72(36)$ & $85(42.5)$ & $200(100)$ & \\
\hline \multicolumn{6}{|c|}{ Mental Distress and Level of Education } \\
\hline Level of Education & \multicolumn{3}{|c|}{ Mental Distress } & \multirow{2}{*}{ Total } & \multirow{2}{*}{ P Value } \\
\hline & Low & Moderate & High & & \\
\hline Primary & $19(21.1)$ & $30(33.3)$ & $41(45.6)$ & $90(45.0)$ & \multirow[b]{2}{*}{$X^{2}=09.904$} \\
\hline Secondary & $22(21.8)$ & $38(37.6)$ & $41(40.6)$ & $101(50.5)$ & \\
\hline Tertiary & $2(22.2)$ & $4(44.4)$ & $3(33.3)$ & $9(4.5)$ & \multirow{2}{*}{$\mathrm{P}<0.924$} \\
\hline Total & $43(21.5)$ & $72(36.0)$ & $85(42.5)$ & $200(100)$ & \\
\hline \multicolumn{6}{|c|}{ Mental Distress and Marital Status } \\
\hline \multirow{2}{*}{ Marital Status } & \multicolumn{3}{|c|}{ Mental Distress } & \multirow{2}{*}{ Total } & \multirow{2}{*}{$P$ Value } \\
\hline & Low & Moderate & High & & \\
\hline Married & $4(16.7)$ & $6(25.0)$ & $14(58.3)$ & $24(12.0)$ & \\
\hline Single & $38(23.4)$ & $58(35.8)$ & $66(40.7)$ & $162(81.0)$ & \multirow{2}{*}{$\mathrm{X}^{2}=7.889$} \\
\hline Divorced & $1(11.1)$ & $4(44.4)$ & $4(44.4)$ & $9(4.5)$ & \\
\hline Widowed & $0(0.0)$ & $4(80.0)$ & $1(20)$ & $5(2.5)$ & \multirow{2}{*}{$\mathrm{P}<0.246$} \\
\hline Total & $43(21.5)$ & $72(36.0)$ & $85(42.5)$ & $200(100)$ & \\
\hline \multicolumn{6}{|c|}{ Mental Distress and Number of Children } \\
\hline Number of Children & & Iental Distr & & Total & P Value \\
\hline Number or Cnilaren & Low & Moderate & High & Total & $P$ Value \\
\hline $1-3$ & $33(23.4)$ & $49(34.7)$ & $59(41.8)$ & $141(70.5)$ & \\
\hline $4-6$ & $8(16.3)$ & $19(38 . .8)$ & $22(44.9)$ & $49(24.5)$ & $\mathrm{X}^{2}=1.164$ \\
\hline Above 6 & $2(20.0)$ & $4(40.0)$ & $4(40.0)$ & $10(5.0)$ & \\
\hline Total & $43(21.5)$ & $72(36.0)$ & $85(42.5)$ & $200(100)$ & $\mathrm{P}<0.884$ \\
\hline & $\mathrm{Me}$ & tress and $R$ & al Type & & \\
\hline Residential Type & & Iental Distr & & Total & P Value \\
\hline & Low & Moderate & High & & \\
\hline Rural & $31(31.9)$ & $37(38.1)$ & $29(29.9)$ & $97(48.5)$ & $X^{2}=16.863$ \\
\hline Urban & $12(11.6)$ & $35(33.9)$ & $56(54.4)$ & $103(51.5)$ & \\
\hline Total & $43(21.5)$ & $72(36.0)$ & $85(42.5)$ & $200(100)$ & $\mathrm{P}<0.001$ \\
\hline & & istress and & ment & & \\
\hline & & Iental Distr & & Total & P Value \\
\hline Confinement Duration & Low & Moderate & High & & \\
\hline Less than 2 weeks & $10(45.4)$ & $8(36.4)$ & $4(18.2)$ & $22(11)$ & \\
\hline 2 weeks to 1 month & $15(18.5)$ & $42(51.8)$ & $24(29.6)$ & $81(40.5)$ & $X^{2}=29.287$ \\
\hline Above 1 month & $18(18.5)$ & $22(22.7)$ & $57(58.8)$ & $97(48.5)$ & $\mathrm{P}<0.001$ \\
\hline Total & $43(21.5)$ & $72(36.0)$ & $85(42.5)$ & $200(100)$ & \\
\hline & Confin & uration an & al Thoughts & & \\
\hline Confinement Duration & & cidal Thoug & & Total & P Value \\
\hline & Hardly Ever & Sometimes & Quite Often & & \\
\hline Less than 2 weeks & $21(95.5)$ & $0(0)$ & $1(4.5)$ & $22(11)$ & \\
\hline 2 weeks to 1 month & $69(85.2)$ & $5(6.2)$ & $5(6.2)$ & $81(40.5)$ & $X^{2}=19.790$ \\
\hline Above 1 month & $59(60.8)$ & $14(14.4)$ & $18(18.5)$ & $97(48.5)$ & $\mathrm{P}<0.001$ \\
\hline Total & $149(74.5)$ & $19(9.5)$ & $24(12)$ & $200(100)$ & \\
\hline
\end{tabular}


In addition, most of the respondents (58.3\%) who were married reported high levels of mental distress compared to 40.7 percent of those who were single, who reported high levels of mental distress (Table 3 ). No statistically significant relationship was found between marital status and mental distress $\left(\mathrm{X}^{2}=7.889, \mathrm{P}<\right.$ 0.246).

Similarly, 41.8 percent of the respondents with less than four children reported high levels of mental distress compared to 40 percent of those with more than six children who also reported high levels of mental distress. Therefore, no statistically significant relationship has been found between respondents' number of children and mental distress $\left(\mathrm{X}^{2}=1.164, \mathrm{P}<0.884\right)$ as depicted in Table 3.

On the other hand, 54.4 percent of respondents who resided in an urban setting reported high levels of mental distress, while 29.9 percent of those who resided in a rural setting reported high levels of mental distress. In addition, 31.9 percent of the respondents residing in the rural setting reported low levels of mental distress, while only 11.6 percent of those who resided in an urban setting reported low levels of mental distress. Therefore, there is a statistically significant relationship between respondents' residential type and mental distress $\left(\mathrm{X}^{2}=\right.$ $16.863, \mathrm{P}<0.001)$.

Accordingly, 58.8 percent of women who were confined for more than a month reported high levels of mental distress in comparison with only 18.2 percent of those who were confined for less than 2 weeks who reported high levels of mental distress. Therefore, there is a statistically significant relationship between duration of confinement and mental distress $\left(\mathrm{X}^{2}=29.287, \mathrm{P}<0.001\right)$.

Further, 18.5 percent of those respondents who were confined for a longer period (over 1 month) admitted having had suicidal thoughts often, while only 6.2 percent and 4.5 percent of those who were confined for a period between two weeks and one month, and less than two weeks experienced suicidal thoughts sometimes and hardly ever, respectively (Table 3 ). There is, therefore a statistically significant relationship between duration of confinement and presence of suicidal thoughts $\left(\mathrm{X}^{2}=19.790, \mathrm{P}<0.003\right)$.

\subsection{Binary Logistic Regression}

From binary logistic regression (Table 4), it was expected that changes in residential type from rural to urban and anxiety from low to high risk would contribute significantly to the regression model while changes in all other variables would not contribute significantly.

On the overall, the regression model was statistically significant $\left(\mathrm{X}^{2}=38.721\right.$, $\mathrm{p}<0.000$ ) and could account for 27.2 percent variation in the outcome variable (Table 4). The model could predict presence of distress with an accuracy of 97.5 percent and absence of distress with 14 percent accuracy. Overall, the model had a prediction accuracy of 79.5 percent.

Inside the model or equation, changes in residential type from rural to urban 
$(\mathrm{p}<0.000$, odds ratio $=0.166)$, marital status from not married to married $(\mathrm{p}<$ 0.013 , odds ratio $=0.231$ ), duration of confinement from less than 2 weeks to between 2 weeks and 1 month $(\mathrm{p}<0.007$, odds ratio $=5.127)$ and from confinement of between 2 weeks and 1 month to over one month ( $\mathrm{p}<0.001$, odds ratio $=7.737$ ) impacted significantly on the model outcome. Changes in all other variable levels did not significantly impact on the outcome of the model, with $\mathrm{p}$-values ranging from 0.086 to 0.852 . Changes in residential type from rural to urban reduced the odds of having mental distress by a multiplicative factor of 0.166. In a similar fashion, changes in marital status from not married to married reduced the odds of experiencing mental distress by a factor of 0.231 . On the other hand, changes in confinement period from below 2 weeks to between 2 weeks and 1 month raised the odds of experiencing mental distress 5.127 times while changes from a confinement period of between 2 weeks and 1 month to over 1 month raised the odds of experiencing mental distress 7.737 times (Table 4).

\section{Discussion}

Perinatal mental distress is relatively common amongst women and occurs in the period from conception to about one year. The present study results reveal a high proportion of women in the postpartum period reporting high levels and

Table 4. Binary logistic regression.

\begin{tabular}{|c|c|c|c|c|c|}
\hline & \multirow{2}{*}{ Variable } & \multirow{2}{*}{ P-Value } & \multirow{2}{*}{ Odds Ratio } & \multicolumn{2}{|c|}{ 95\% C.I } \\
\hline & & & & Lower & Upper \\
\hline & Residential Type (1) & 0.000 & 0.166 & 0.064 & 0.433 \\
\hline & Age & 0.434 & & & \\
\hline & Age (1) & 0.450 & 0.662 & 0.227 & 1.929 \\
\hline & Age (2) & 0.388 & 1.668 & 0.522 & 5.331 \\
\hline & Age (3) & 0.848 & 1.133 & 0.315 & 4.079 \\
\hline & Education & 0.852 & & & \\
\hline & Education (1) & 0.813 & 1.101 & 0.495 & 2.450 \\
\hline & Education (2) & 0.658 & 0.646 & 0.094 & 4.467 \\
\hline Step $1^{\mathrm{a}}$ & Mstatus (1) & 0.013 & 0.231 & 0.073 & 0.736 \\
\hline & Parity & 0.686 & & & \\
\hline & Parity (1) & 0.495 & 1.433 & 0.510 & 4.027 \\
\hline & Parity (2) & 0.520 & 1.788 & 0.305 & 10.484 \\
\hline & Confinement & 0.004 & & & \\
\hline & Confinement (1) & 0.007 & 5.127 & 1.563 & 16.811 \\
\hline & Confinement (2) & 0.001 & 7.737 & 2.293 & 26.102 \\
\hline & Anxiety (1) & 0.086 & 4.026 & 0.822 & 19.719 \\
\hline & Constant & 0.037 & 5.083 & & \\
\hline
\end{tabular}


moderate levels mental distress ( 44.5 and 36 percent respectively). These findings corroborate with Norhayati et al.'s [21] update which reveals the prevalence as estimated to fall between 1.9 percent and 82.1 percent in low-income countries, and a record prevalence of between 5.2 percent and 74.0 percent high-income countries. The prevalence for both low-income and high-income countries has been found to be higher than that earlier reported, albeit with similar associated factors [21].

The high prevalence may be attributed to a number of factors which may include unrealistic expectations on the part of women, and a lack of support, among others. Additional pressures such as a traumatic birth, financial or relationship problems or an unsettled baby have also been implicated. Cultural and traditional practices involving confinement following childbirth, a traditional practice designed to allow postnatal women some physical and emotional rest, may escalate women's vulnerability to postnatal mental distress [21].

Therefore, the present study whose aim was to explore women's confinement following childbirth and its association with postpartum mental distress will serve to inform policy and clinical practice, and catalyse policy development and implementation that responds to the needs of postpartum women. It will also augment support for the development of interventions that target minimizing women's vulnerability to postpartum mental distress associated with confinement after childbirth.

The findings reveal a statistically significant relationship between mental distress and the age of the participants, and mental distress and residential type (rural or urban) with Chi square and $\mathrm{P}$ values of $\mathrm{X}^{2}=17.996, \mathrm{P}<0.006 ; \mathrm{X}^{2}=$ 16.863, $\mathrm{P}<0.001$ respectively. The results support Aasheim et al., [22] and Giri et al's [23] findings which also revealed a relationship between mental distress and age, divergent from Clarke et al., [24] who recorded no association between mental distress and age. For residential area and mental distress, the results of the present study corroborate with Pattyn et al., [25] whose findings revealed a statistically significant relationship between residential area and mental distress, recording higher prevalence of mental distress in the population from high density, economically constrained areas compared to low density, higher income areas.

Surprisingly, level of education, marital status, and parity have been found to have no statistically significant relationship with mental distress in the present study $\left(\mathrm{X}^{2}=09.904, \mathrm{P}<0.924 ; \mathrm{X}^{2}=7.889, \mathrm{P}<0.246 ; \mathrm{X}^{2}=1.164, \mathrm{P}=0.884\right.$ respectively). Contrary to these findings, Pattyn et al., [25] and Clarke et al., [24] reveal more complaints of psychological distress by women with low education level, and Qadir et al., [26] emphasizes that education is viewed as a protective element against mental distress because it shapes attitudes, lifestyle and behaviors, which may enhance the mental health of women. Marriage in many societies is considered an essential part of life and therefore, it is not uncommon to find no relationship between mental distress and marital status, especially given that in some cultures including Zambia, marriage is a means of support for women, and 
in this case serves as a protective factor against postpartum mental distress, although it is argued that women are more vulnerable to mental distress arising from marriage compared to their male counterparts [27].

\subsection{Mental Distress and Confinement}

Postpartum mental distress leading to depression is a common cause of disease-related morbidity among women [28], but it is undertreated in many low and middle-income countries, especially sub-Saharan Africa [29]. While culture influences the social determinants and expression of symptoms of postpartum mental distress, there is paucity of research studies on this subject in African contexts [30].

For women of childbearing age, postpartum mental distress has been cited to be second after HIV/AIDS in total disability [30]. The present study findings reveal a relationship between confinement, and postpartum mental distress $\left(\mathrm{X}^{2}=\right.$ 19.790; P < 0.001). Norhayati, et al., [21] concur with this finding and state that traditional confinement following child birth is one of the contributing factors to postpartum mental distress.

It is revealed that a negative confinement experience was associated with postpartum mental distress. Poor emotional support, history of depression, unplanned pregnancy and perceived potential conflicts with relatives over childcare, and inadequate support were additional factors that were found to contribute to postpartum mental distress [31].

Further, in Singapore, confinement has been found to be a significant risk factor for postpartum mental distress, and is not generally welcomed by women [31]. On the contrary, the Chinese postpartum custom of "confinement" or "doing-the-month" as it is commonly known, involves formal social support and appreciation of the significance of motherhood and has been presumed to be a protective sheath for mothers of newborns from postpartum depression [32].

However, Chee et al., [31] argue that the complexity of the role of confinement in postpartum mental distress should not be overestimated while Sharma et al., [33] acknowledges that presence of psychological distress during the postnatal period may be associated with postnatal traditional practices. The review highlighted that while some studies found confinement to be a protective factor; others have found it to perpetuate the vulnerability to postpartum mood disturbance. As such, elements of the confinement practice that might contribute to postpartum mental distress may include diminished social support, conflict with a mother-in-law, and the tension experienced by modern women as they work to balance traditional with contemporary values.

As highlighted above, Wong and Fisher [32], found little concrete evidence pointing to confinement practices as protective factors against postpartum distress in Chinese cultures. Although specific components of confinement practices might reduce psychological distress in Chinese mothers of newborns, these cannot be differentiated from the existing evidence and therefore, confinement cannot be presumed to be available to, welcomed by or effective for all Chinese 
women or to be a substitute for health care services.

Women appreciated an isolated period of postnatal confinement of up to 3 months, and they recognized rest and recovery, and their newly acquired status, as the shared sociocultural patterning of the postnatal period. On the other hand, women identified situations which might serve as contributing factors to postpartum distress as including "disappointed expectations and exclusion", "exacerbating pre-existing problems" and "vulnerability and danger" regardless of their socioeconomic status [34]. Klainin and Arthur's [35] study further highlight that traditional postpartum confinement practices were found inadequate with regards to providing significant psychological benefits for postnatal women.

Failure by women to conform to postpartum traditional practices, in itself has also been reported as a factor contributing to the risk of postpartum mental distress due to the stress that accompanies non conformity. On the other hand, the rituals performed during postpartum confinement add to women's distress, which in some cases may be linked to suicidal thoughts [29].

However, Demiriel et al. [4], argue that traditional practices in general and confinement in particular, may act as a buffer to mental distress for some women, while on the other hand, the same traditional practices may serve to perpetuate mental distress, in other women [4]. The dearth of literature in this area may explain the divergent opinions among researchers, and therefore, the need for further studies should be given attention [4]. In addition, the difference in the period of confinement as well as the type of rituals performed in different parts of the world may also determine vulnerability to postpartum mental distress. Hence, health care should be receptive to traditional practices as they provide care to women in the postpartum period in order to reduce the risk of mental distress while looking out for those who may at risk [33].

\subsection{Postpartum Confinement and Suicidal Thoughts}

Suicide is one of the leading causes of death in postpartum women [36], [37]. The present study found a statistically significant relationship between confinement and suicidal thoughts $\left(\mathrm{X}^{2}=19.790 ; \mathrm{P}<0.001\right)$. These suicidal thoughts may be as a result of the mental distress experienced by the population under study $\left(\mathrm{X}^{2}=29.287 ; \mathrm{P}<0.001\right)$. It is documented that suicide is the seventh leading cause of maternal death within six months of delivery with an estimated 1.27 per 100,000 maternal deaths [38]. Therefore, women in postpartum confinement during this period can present with signs of mental distress giving rise frequent thoughts of self-harm. Identifying modifiable factors related to suicide risk in mothers after delivery should be a public health priority. Wisner et al., [36] report that about 85 percent of women in their study who scored above the cut-off point on the EPDS had also contemplated suicide.

\section{Strengths and Limitations}

With the paucity of literature in the area of maternal mental health in Zambia, this study is one of the few, if any, to delve into mental distress that might arise 
from the traditional practice of confinement following childbirth. The study had representation from the urban settings as well as the rural settings. Although the study confirmed the association between confinement and mental distress, restricting the study to only two districts in two provinces of the country is one of the limitations. It is possible that inclusion of more districts that might practice confinement differently may have yielded different insights. Nevertheless, the study has provided results that form the foundation on which to build future studies in this area of health.

\section{Conclusion}

Confinement following childbirth is a tradition widely practiced in many cultures around the world. The period of confinement and the activities performed during the period of confinement differ from culture to culture and from society to society. This study results reveal the presence of mental distress during the postnatal period in the study population. The study findings revealed that the longer the period of postpartum confinement, the more likely it is for the woman to experience mental distress, and the more likely it is for women to experience suicidal thoughts. Therefore, deliberate efforts should be made by health care providers, especially nurses and midwives to screen women during the postnatal period in general, and those in confinement in particular, for potential risk. Early identification should target postpartum women and especially those women in confinement, so that appropriate interventions are initiated timely. Scaling up awareness and sensitization efforts and campaigns to integrate mental health into main stream health services may also minimize the risk of mental distress during the postnatal period.

\section{Acknowledgements}

We wish to acknowledge the financial support provided by the Agency for Development (NORAD) through the NORHED QZA-0848 QZAMW-13/00032 Grant.

\section{References}

[1] Okka, B., Durduran, Y. and Degerli Kodaz, N. (2016) Traditional Practices of Konya Women during Pregnancy, Birth, the Postpartum Period, and Newborn. Turkish Journal of Medical Sciences, 46, 501-511. https://doi.org/10.3906/sag-1504-120

[2] World Health Organization (2013) World Health Organization Recommendations on Postnatal Care of the Mother and Newborn. World Health Organization, Geneva.

[3] Baby Centre (2014) Confinement Practices: An Overview. http://www.babycentre.com

[4] Demirel, G., Egri, G., Yesildag, B. and Doganer, A. (2018) Effects of Traditional Practices in the Postpartum Period on Postpartum Depression. Health Care Women International, 39, 65-78. https://doi.org/10.1080/07399332.2017.1370469

[5] Dennis, C.L., Fung, K., Grigoriadis, S., Robinson, G.E., Romans, S. and Ross, L. 
(2007) Traditional Postpartum Practices and Rituals: A Qualitative Systematic Review. Women's Health, 3, 487-502. https://doi.org/10.2217/17455057.3.4.487

[6] Cousik, R. and Hickey, M. (2016) Pregnancy and Childbirth Practices among Immigrant Women from India: "Have a Healthy Baby". The Qualitative Report, 21, 727-743.

[7] Chien, L.Y., Chen, J.T., Ko, Y.L., Huang, C.H. and Sheu, S.J. (2006) Adherence to Doing-the-Month Practices Is Associated with Better Health Status in Women in Taiwan. Research in Nursing and Health, 29, 374-383. https://doi.org/10.1002/nur.20154

[8] Chen, T.L., Tai, C.J., Wu, T.W., Chiang, C.P. and Chien, L.Y. (2012) Postpartum Cultural Practices Are Negatively Associated with Depressive Symptoms among Chinese and Vietnamese Mothers Married to Taiwanese Men. Women \& Health, 52, 536-562. https://doi.org/10.1080/03630242.2012.697109

[9] Leung, S.K., Arthur, D. and Martinson, I.M. (2005) Perceived Stress and Support of the Chinese Postpartum Ritual "Doing-the-Month". Health Care Women International, 26, 212-224. https://doi.org/10.1080/07399330590917771

[10] Liu, Y.Q., Maloni, J.A. and Petrini, M.A. (2012) Effect of Postpartum Practices of Doing the Month on Chinese Women's Physical and Psychological Health. Biological Research for Nursing, 16, 55-63. https://doi.org/10.1177/1099800412465107

[11] Abdollahi, F., Lye, M.S., Md Zain, A., Shariff Ghazali, S. and Zarghami, M. (2011) Postnatal Depression and Its Associated Factors in Women from Different Cultures. Iranian Journal of Psychiatry and Behavioral Sciences, 5, 5-11.

[12] Yeh, Y.C., St. John, W. and Venturato, L. (2013) Doing the Month in the Taiwanese Postpartum Nursing Centre: An Ethnographic Study. Nursing and Health Sciences, 16, 343-351. https://doi.org/10.1111/nhs.12110

[13] Schiller, C.E., Melzer-Brody, S. and Rubinow, D.R. (2015) The Role of Reproductive Hormones in Postpartum Depression. CNS Spectrums, 20, 48-59. https://doi.org/10.1017/S1092852914000480

[14] Dobson, B. and Sales, B. (2000) The Science of Infanticide and Mental Illness. Psychology, Public Policy, and Law, 6, 1098-1112. https://doi.org/10.1037/1076-8971.6.4.1098

[15] Howard, L.M., Molyneaux, E., Dennis, C.-L., Rochat, T., Stein, A. and Milgrom, J. (2014) Non-Psychotic Mental Disorders in the Perinatal Period. The Lancet, 384, 1775-1788. https://doi.org/10.1016/S0140-6736(14)61276-9

[16] Stein, A., Pearson, R.M., Goodman, S.H., Rapa, E., Rahman, A., McCallum, M.L.M. and Pariante, C.M. (2014) Effects of Perinatal Mental Disorders on the Foetus and Child. The Lancet, 384, 1800-1819. https://doi.org/10.1016/S0140-6736(14)61277-0

[17] Polit, D.F. and Beck, C.T. (2012) Nursing Research; Generating and Assessing Evidence for Nursing Practice. Lippincot Williams \& Wilkins, Philadelphia.

[18] Lemeshow, S.H., Klar, D.W., Lwanga, J., Kaggwa, S. and World Health Organisation (1990) Adequacy of Sample Size in Health Studies. Wiley, Chichester.

[19] Cox, J.L. (1999) Perinatal Mood Disorders in a Changing Culture. A Transcultural European and African Perspective. International Review of Psychiatry, 11, 103-110. https://doi.org/10.1080/09540269974258

[20] Cox, J.L., Holden, J.M. and Sagovsky, R. (1987) Detection of Postnatal Depression. Development of the 10-Item Edinburgh Postnatal Depression Scale. British Journal of Psychiatry, 150, 782-786. https://doi.org/10.1192/bjp.150.6.782

[21] Norhayati, M.N., Nikhalina, N.H., Asrenee, A.R. and Wan Emilin, W.M.A. (2015) 
Magnitude and Risk Factors for Postnatal Symptoms: A Literature Review. Journal of Affective Disorders, 175, 34-52. https://doi.org/10.1016/j.jad.2014.12.041

[22] Aasheim, V., Waldenstro, U., Hjelmstedt, A., Rasmussen, S., Pettersen, H. and Schytt, E. (2012) Associations between Advanced Maternal Age and Psychological Distress in Primiparous Women, from Early Pregnancy to 18 Months Postpartum. British Journal of Obstetrics \& Gynaecology, 119, 1108-1116. https://doi.org/10.1111/j.1471-0528.2012.03411.x

[23] Giri, R.K., Khatri, R.B., Mishra, S.R., Khanai, V., Sharma, V.D. and Gartoula, R.P. (2015) Prevalence and Factors Associated with Depressive Symptoms among Postpartum Mother in Nepal. BMC Research Notes, 8, 111. https://doi.org/10.1186/s13104-015-1074-3

[24] Clarke, K., Saville, N., Shrestha, B., Costello, A., King, M., Manandhar, D.O. and Prost, A. (2014) Predictors of Psychological Distress among Mothers in Rural Nepal: A Cross-Sectional Community Based Study. Journal of Affective Disorders, 156, 76-86. https://doi.org/10.1016/j.jad.2013.11.018

[25] Pattyn, E., Van Praag, L., Verhaeghe, M., Levecque, K. and Bracke, P. (2011) The Association between Residential Area Characteristics and Mental Health Outcomes among Men and Women in Belgium. Archives of Public Health, 69, 13. https://doi.org/10.1186/0778-7367-69-3

[26] Qadir, F., Khan, M.M., Medhin, G. and Prince, M. (2011) Male Gender Preference, Female Gender Disadvantage as Risk Factors for Psychological Morbidity in Pakistani Women of Childbearing Age-A Life Course Perspective. BMC Public Health, 11, 745. https://doi.org/10.1186/1471-2458-11-745

[27] Wishman, M.A., Weinstock, L.M. and Tolejko, N. (2006) Marriage and Depression. In: Keyes, C.L.M. and Goodman, S.H., Eds., Women and Depression: A Handbook for the Social, Behavioral, and Biomedical Sciences, Cambridge University Press, New York, 219-240. https://doi.org/10.1017/CBO9780511841262.012

[28] Kessler, R.C. (2003) Epidemiology of Women and Depression. Journal of Affective Disorders, 74, 5-13. https://doi.org/10.1016/S0165-0327(02)00426-3

[29] Adeponle, A., Groleau, D., Kola, L., Kirmayer, L.J. and Gureje, O. (2017) Perinatal Depression in Nigeria: Perspectives of Women, Family Caregivers and Health Care Providers. International Journal of Mental Health Systems, 11, 27. https://doi.org/10.1186/s13033-017-0134-6

[30] World Health Organization (2001) Mental Health: New Understanding, New Hope. World Health Report, World Health Organization, Geneva.

[31] Chee, C.Y.I., Lee, D.T.S., Chong, Y.S., Tan, L.K., Ng, T.P. and Calvin, S.L. (2005) Confinement and Other Psychosocial Factors in Perinatal Depression: A Transcultural Study in Singapore. Journal of Affective Disorders, 89, 157-166. https://doi.org/10.1016/j.jad.2005.09.004

[32] Wong, J. and Fisher, J. (2009) The Role of Traditional Confinement Practices in Determining Postpartum Depression in Women in Chinese Cultures: A Systematic Review of the English Language Evidence. Journal of Affective Disorders, 116, 161-169. https://doi.org/10.1016/j.jad.2008.11.002

[33] Sharma, V., Doobay, M. and Baczynski, C. (2017) Bipolar Postpartum Depression: An Update and Recommendations. Journal of Affective Disorders, 219, 105-111. https://doi.org/10.1016/j.jad.2017.05.014

[34] Hanlon, C., Whitley, R., Wondimagegn, D., Alem, A. and Prince, M. (2009) Postnatal Mental Distress in relation to the Sociocultural Practices of Childbirth: An Exploratory Qualitative Study from Ethiopia. Social Sciences \& Medicine, 69, 
1211-1219. https://doi.org/10.1016/j.socscimed.2009.07.043

[35] Klainin, P. and Arthur, G. (2009) Postpartum Depression in Asian Cultures: A Literature Review. International Journal of Nursing Standards, 46, 1355-1373. https://doi.org/10.1016/j.ijnurstu.2009.02.012

[36] Wisner, K.L., Sit, D.Y., McShea, M.C., Rizz, D.M., Zoretich, R.A., Hughes, C.L, Eng, H.F., Luther, J.F., Wisniewski, S.R., Costantino, M.L., Confer, A.L., Moses-Kalko, E.L., Famy, C.S. and Hanusa, B.H. (2013) Onset Timing, Thoughts of Self-Harm, and Diagnoses in Postpartum Women with Screen-Positive Depression Findings. JAMA Psychiatry, 70, 490-498. https://doi.org/10.1001/jamapsychiatry.2013.87

[37] Oates, M. (2003) Perinatal Psychiatric Disorders: A Leading Cause of Maternal Morbidity and Mortality. British Medical Bulleting, 67, 219-229.

https://doi.org/10.1093/bmb/ldg011

[38] Lewis, G., Cantwell, R., Clutton-Brock, T., Cooper, G., Dawson, A., Drife, J.D., Garrod, D., Harper, A., Hulbert, D., Lucas, S., McClure, J., Millward-Sadler, H., Neilson, J., Nelson-Piercy, C., Norman, J., O’Herlihy, C., Oates, M., Shakespeare, J., de Swiet, M., Williamson, C., Beale, V., Knight, M., Lennox, C., Miller, A., Parmar, D., Rogers, J. and Springett, A. (2011) Saving Mothers' Lives: Reviewing Maternal Deaths to Make Motherhood Safer. International Journal Obstetrics \& Gynaecology, 118, 1-203. https://doi.org/10.1111/j.1471-0528.2010.02847.x 